\title{
Italique
}

Poésie italienne de la Renaissance

II | 1999

Varia

\section{Note per un bestiario lirico tra quattro e cinquecento}

Massimo Malinverni

\section{(2) OpenEdition}

Edizione digitale

URL: https://journals.openedition.org/italique/67

DOI: 10.4000/italique.67

ISSN: 1663-4438

\section{Editore}

Librairie Droz

\section{Edizione cartacea}

Data di pubblicazione: 1 décembre 1999

Paginazione: 7-31

ISBN: 2-600-00389-4

ISSN: 1423-3983

Notizia bibliografica digitale

Massimo Malinverni, «Note per un bestiario lirico tra quattro e cinquecento», Italique [Online], II | 1999, online dal 07 octobre 2009, consultato il 26 novembre 2022. URL: http://journals.openedition.org/ italique/67 ; DOI: https://doi.org/10.4000/italique.67

All rights reserved 
MAS S I O MALIN V ER N I

NOTE PER UN B ESTIARIO LIRICO

TRA Q U A T TRO E C I N Q U E C E N T O 


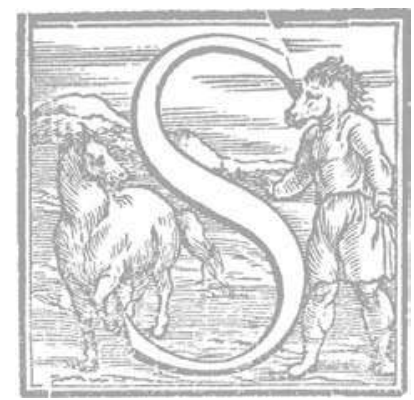

E è vero (come scrive Francesco Zambon nell'introdurione alla sua versione italiana del Physiologus) ${ }^{\mathrm{I}}$ che attraverso gli animali l'uomo ha creduto in passato di leggere o fingere, per speculum in aenigmate, verità metafisiche non altrimenti attingibili, per $i$ rimatori volgari dei primi secoli varrà almeno, sub specie più cauta ed immanente, una suggestione continua, etica insieme e figurativa, al confronto ravvicinato con i nostri compagni di pena, o gioia, terrestre: quasi a voler quotidianamente verificare, al di là del ricorso stilizzato a topoi antichissimi, un radicamento originario e rassicurante a pulsioni, comportamenti e passioni sorprendentemente comuni. Senza contare il fascino costante e non estinguibile, nei secoli, del simbolo morale, della figura come ancoramento sintetico e potente per il nostro procedere così raramente, e fondatamente, orientato. Ed è allora facile prevedere quanto frequente, consentaneo e talvolta ossessivo divenga il ricorso ad immagini e tipi del mondo animale nell'espressiva e talvolta incontinente stagione lirica di fine Quattrocento. Segnatamente nella variante, più impaziente di canoni e monogame fedeltà imitative, dei cosiddetti «cortigiani », ${ }^{2}$ particolarmente fascinati dagli spunti rappresentativi più vivaci ed icastici. Da qui lo spunto per le poche Note, o meglio schede essenziali, che seguono. Tutte riguardanti, principalmente per assidua frequentazione di editore in fieri, il canzoniere di Panfilo Sasso: ${ }^{3}$ probabilmente, ad ogni buon conto, ilpiù sbilanciato fra gli autori del tempo in un' accesapredilezione per l'exemplum naturalistico e animale. Valgano comunque, a parziale raffronto, almeno le sparse ma utilissime indicazioni desumibili dallo studio di Antonio Rossi sull' Aquilano, ${ }^{4}$ come dalla ricchissima annotazione di Tania Basile al Tebaldeo.' Con la doverosa avvertenza, nel caso presente, di non aver minimamente teso ad un'impossibile esaustività dei riscontri di tradizione, nétantomeno di aver voluto ambire ad una vera e propria storia del genere "bestiario » nella sua sottospecie lirica: genere, ad immediata conferma, non sempre strettamente rispettato, in sede di analisi, a favore di comportamenti e figure propriamente extracanonici (escludendo, comunque, gli accenni generici ad animali che non svolgano un ruolo esemplarmente rilevato ed attivo).

Lo sparviero e il leone magnanimi, il gallo e la rana pusillanimi

D'un tristo ucel se sdegna el sparavero, ben ch'abia fame, pigliar nutrimento; non fa preda el leon d'un vil armento, ma d'un qualche animal superbo e fero. 


\title{
Massimo MaLinverni
}

\author{
Contra el vilan l'ardito cavalero \\ non piglia l'arme, a la sua gloria intento; \\ quanto è magior fortuna e più fier vento \\ tanto più scorre in alto el bon nochiero. \\ A degna impresa un cor degno e soprano \\ driza el sò corso. Tu come el gal fai, \\ lassi la giemma per beccar el grano. \\ Dir m'el convien perché sforzato m'hai : \\ non fu chi fòr la rana del pantano \\ in alcun modo trar potesse mai.
}

Agli esempi di magnanimitas, particolarmente vulgati, dei Vv. I-IO sono contrapposti in chiusura due esempi altrettanto tradizionali di comportamento pusillanime (questa volta esclusivamente dal mondo animale), a stigmatizzare sdegnosamente il tradimento della donna con un servo vile (cfr. son. 48, 9-II : "Serviva como un cane incatenato, / e quella che m'havea ligato al laccio / m'ha per un servo vile abandonato »).

Il falcone (in questo caso sparavero, sparviero) e il leone sono già accostati come modelli di magnanimità nel bestiario "moralizzato» del Fiore di virtù, cap. XXVIII (citiamo dall'edir. di Venezia, nel Beretin convento de la cha grande, I474) : "E puose apropriare e asemîare la virtù del Magnanimità al Falcone: lo se lasarave inanci morire da fame che lo manzase de una carne marża, e non pîa mai se non oselli grossi. Sancto Augustino dice: lo Lione non fa guerra cum le formiche e l'Aquila non pîa mosche ». Dalla stessa tradizione derivano anche CECCO D'AsCOLI, Acerba 2448-49: "Non becca mai della putrida carne, / sia quanto vuole di fame converso» $e$ LEONARDO, Bestiario 23: "Magnanimità. Il falcone non preda mai se non e uccelli grossi, e prima si lascerebbe morire che si cibassi de' piccoli o che mangiasse carne fetida 》. E cfr., in ambito lirico, GALLO I II3, 5-6: "Un falcon singulare e pellegrino / a vil preda calarsi arebbe a sdegno ».

L'apologo del gallo (vv. IO-II) che nel cercare del cibo in un letamaio scopre invece una gemma, e la disprezza in quanto inutile a soddisfare la sua fame, $\grave{e}$ in FEDRO (Pullus ad margaritam, III I2), ma fino alla princeps del I596 era noto soltanto attraverso l'eterogeneo corpus delle parafrasi pseudoesopiche : in particolare, nel Quattrocento, quella in distici di Gualtiero Anglico (XII sec.) : cfr. De gallo et iaspide, in Aesopus moralisatus, Verona, Giovanni e Alberto Alvise, I479, cc. asv-abr. In quanto al particolare del grano, assente nell'originale (dove si parla genericamente di escam), è probabilmente derivato dalla versione volgare trecentesca di ACCIO $Z_{U C C O}$, immediatamente fatta seguire, in questa ed altre edizioni quattrocentesche, al 
testo latino : "Dice il maestro che 'l gallo, raspando / dentro al letame per trovar del grano, / maravigliosse che gli venne a mano / una preciosa pietra [...] ». Per l'espressione, di chiara origine proverbiale, dei vv. I3-I4 cfr. ad es. ANTONIO dI MEGLIO, XIII 32-33 (Lirici toscani II 9I) : "Perde tempo in chiamarla / la rana, chi vuol trarla - del pantano »; e la rubrica del son. del FELICLANO, Tenne gran tempo tra le griffe un gatto (cito dal ms. a.H.6.I [ital. 836] della Biblioteca Estense di Modena, c. 43r): " Foeliciano affirma esser impossibil cosa trar la rana del pantano, et torcer la persona del suo naturale ».

Il sordo aspe

L'incanto fa il sordo aspe obediente, rompe le dure pietre, abassa i monti ; l'incanto ferma i fiumi e secca i fonti, obscura el sol d'istade in oriente.

L'incanto el mar turbato fa la gente passar sencia ale, sencia nave e ponti; l'incanto fa doi tigri, insieme agionti, portar el duro giogo humanamente.

L'incanto pò dal ciel tirar la luna, l'antiqua etade a la nova tornare; l'incanto vince Amore et la Fortuna, et non pò la tua mente, aspra, mutare : unde passi di orgolio, in el mondo una, monti, fiumi, aspi, tigri, pietre e mare.

La donna, come dichiara l'incipit del successivo son. 242 (a questo strettamente legato : vedi più sotto) è fatta un aspe sordo al dolce incanto dei versi del poeta, e viene proposta come esempio supremo e singolare di orgoglio (ossia adamantina indifferen₹a, nonché ai sentimenti dell' amante, alla stessa magia dell'incanto) nel confronto con ogni animale od elemento naturale.

Oviamente diffusissima è l'immagine dell'incantamento dei serpenti : cfr. in particolare TIBULLO I VIII Ig ss. (probabile ispiratore della presente struttura anaforica) : "Cantus vicinis fruges traducit ab agris, / cantus et iratae detinet anguis iter, / cantus et e curru Lunam deducere temptat »; quindi VIRGILIO, Aen. VII 753-55; OVIDIO, Met. VII 203; SENECA, Medea 684-90; LUCANO, Phars. VI 488-9I ; infine, per la sottolineatura del potere della poesia, Rvf. 239, 28-30: "Nulla al mondo è che non possano i versi : / 
et li aspidi incantar sanno in lor note, / nonché 'l gielo adornar di novi fiori》; e cfr. anche BOLARDO, A. L. CXI I-2: "Qual si move constretto da la fede / de' tesalici incanti il frigido angue ». Ma per sordo aspe $\grave{e}$ fondamentale l'archetipo scritturale di Ps 57, 5-6 (" sicut aspidis surdae et obturantis aures suas / quae non exaudiet vocem incantantium et veneficii incantantis sapienter »), in seguito ripreso da un'amplissima tradizione leggendaria medioevale (cfr., per tutti, ISIDORO, Etym. XII IV I2 : "Fertur autem aspis, cum coeperit pati incantatorem, qui eam quibusdam carminibus propriis evocat ut eam de caverna producat : illa, cum exire noluerit, unam aurem in terram premit, alteram cauda obturat et operit, atque [ita] voces illas magicas non audiens non exit ad incantantem 》) ed ovviamente volgare: si vedano almeno, in poesia, Rvf. 210, $7:$ " che sol trovo Pietà sorda com'aspe »; CECCO D'AsCOLI, Acerba 2652-53: «Per non sentire la magica prece, / ciascuna orecchia ottura e tien coperta »; PULCI, Morgante XIV 83, $5:$ "l'aspido sordo, freddo più che lastra 》 (e ancora, pur con sourasenso osceno, MaCHLavelLI, Canti carn. VI I9: "L'aspido sordo è un tristo animale »). Mentre GIUSTo LX I-4 ("O Dio, ch'al vento perdo le parole, / et cerco l'orso bumiliar co 'l pianto, / misero, con la morte allato incanto / l'aspido sordo che ascoltar non vuole ») è il preciso riferimento per il luogo parallelo di son. 242, I-4:

Sei fatta un aspe sordo al dolce incanto

d'i versi mei, che verso alcun non cura;

sei fatta un'orsa dispietata e dura

a' mei sospir', a' mei lamenti, al pianto.

Meno precisamente connotata è invece l'occorrenza del sintagma nell'incipit del son. 79 :

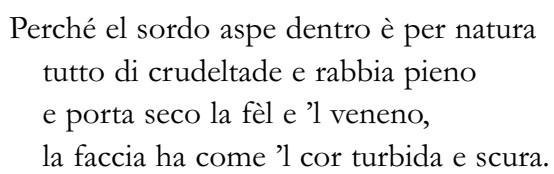

Le due tigri

A fronte di un generico accenno al singolare in son. 79, 5-6 ("El tigre fa con el guardo paura / perché è spietato [...] » : normale a questa altezza la forma maschile) è invece degna di nota la persistenza in due luoghi dell'immagine dei 
dui tigri, pur con diversa fun₹ionalità semantica ed espressiva. In son. I26, 5-8:

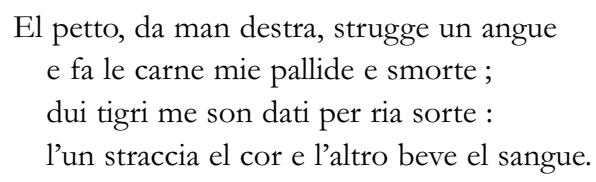

prevale l'intento di assimilazione icastica ad un contesto iperbolicamente " disperato 》. Mentre in son. 24I, 7-8, come si è già visto ("l'incanto fa doi tigri, insieme agionti, / portar el duro giogo humanamente »), nonostante l'accentuazione, quasi da mundus inversus, qui operata (le due tigri unite [agionti] nel portare pacificamente [humanamente] il giogo come una coppia di buoi), è pur frequente nei classici l'immagine della tigre ammansita, certo meno per opera dell'arte magica (cfr. LUCANO, Phars. VI 487-88: "Has avidae tigres et nobilis ira leonum / ore fovent blando ") che del potere fascinatorio del canto e della poesia : cfr. VIRGILIO, Georg. IV sIO (" mulcentem tigris »: da cui di nuovo SASSO, De morte Angeli Politiani, 9 : «mulcebat tigres»); ORAZIO, Carm. III XI I3-I4 e Ars. poet. 393; ma soprattutto CLaUdLano, De raptu Pros. Prol. l. II $27:$ : Concordes varia ludunt cum tigride dammae» (da cui Polizlano, Orfeo I63 : "la cervia e'l tigre insieme havemo accolti »).

\section{Il cervo}

Diverse sono le pur topiche rappresentazioni della natura del cervo esperite in altrettanto differenti contesti, spesso in funzione di figurante dell'esperienza amorosa del poeta. Dal semplice quadro naturalistico di son. I39, I-4:

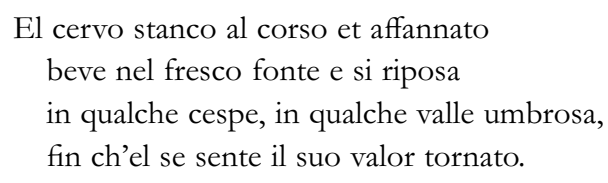

e dall'acuto schizzo figurativo di un tipico atteggiamento dell'animale (son. 239, 3, all'interno di un elenco di comportamenti di impossibile imitazione):

\footnotetext{
Chi fede pensa fida in hom mortale trovar, pensa trar fòr del piumbo argento, como el cervo saltar cum passo lento e volar più d'ucel non havendo ale,
} 


\section{Massimo MaLinverni}

si passa, in altre due occorrenze, ad una più intensa assunzione metaforica ed esemplare, quasi sempre sulla scorta di precedenti classici e volgari. Nel son. I22 :

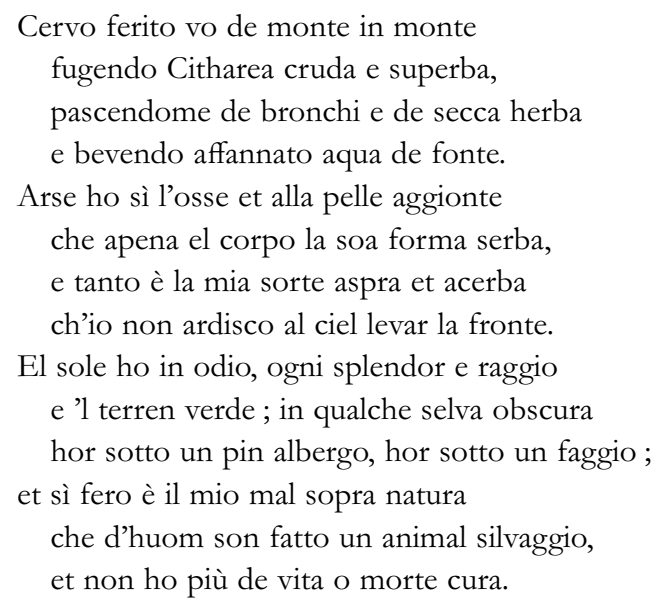

all'interno di una serie di testi sul tema della fuga del poeta disperato e quasi reso selvatico dalle pene amorose in "logi inculti, horrendi et atri», lontani dalla vita civile e da ogni luogo abitato, si assiste nell'incipit alla fusione di tre ricordi petrarcheschi: Rvf. 209, 9 ("Et qual cervo ferito di saetta » : con il passaggio dall'analogia esplicita, introdotta da " qual» e direttamente risalente al modello virgiliano di Aen. IV 60 : " [...] qualis coniecta cerva sagitta », all'assunzione metaforica diretta in sede iniziale, con evidente effetto di intensificazione simbolica); I29, I ("Di pensier in pensier, di monte in monte »: e cfr. anche, al v. 4 e sempre in rima, "fonte » di I29, 4); con in più una sorta di calco ritmico-tonale, certo drammaticamente ed iperbolicamente accentuato, di 35, I ss. ("Solo et pensoso i più deserti campi / vo mesurando... »).

Mentre nel son. I80:

Timido, afflitto, lasso e doloroso non perché fallito habia como el servo che è stato contra al suo signor protervo, vengo denanci al tuo seggio famoso;

ma perché al primo tuo guardo amoroso ogni mio senso, ogni mio polso e nervo como Diana tramutasti in cervo : ond'io, fatto veloce et pauroso, per non esser da' cani empii stracciato, 
che già me cominciaveno a ferire aciò ch'io fusse del tutto impiagato, presi consiglio de dover fugire.

Et hor sotto el tuo stral son ritornato, stimando più che vita un bel morire.

il ricordo, ai vv. 5-I2, della morte di Atteone mutato in cervo da Diana e quindi straziato dai cani (cfr. OVIDIO, Met. III I37 ss.) è probabilmente mediato dalla «metamorfosi » petrarchesca di Rvf. 23, I47-60 (cfr. almeno $i$ $v v$. I58-60: "et in un cervo solitario et vago / di selva in selva ratto mi trasformo : / et anchor de' miei can' fuggo lo stormo »).

\section{La fenice}

L'immagine della fenice eternamente rinascente dalle proprie ceneri (cfr., dall'antichità, almeno ERODOTO II 73 ; PLINIO, Nat. hist. X 2, 3 ; Phys. 7), quindi esempio moralizzato di "Constanzia 》 (ancora secondo l'intitolazione di LEONARDO, Bestiario 25; in ambito lirico vedi CHLARO DAVANZATI XXIII I9-22 e 24, I-4; Mare amoroso 270-7I [Poeti '200 I 497]; quindi CECCO D'ASCOLI, Acerba 2075-86), è prevalentemente usufruita dal Sasso ${ }^{6}$ come simbolo e contrario della propria infelice condizione amorosa. In son. I27, 9-I4:

Apparecchiato ho già l'ultime fasce per far nel foco come la phenice, e già la fiamma el tristo cor mi pasce.

Lei fortunata mòr, como se dice ; ma tristo me se 'l mio spirto rinasce, ché naque per non esser mai felice.

è evidente la mediazione dantesca di Inf. XXIV III : " e nardo e mirra son l'ultime fasce » (da cui anche le altre parole-rima «pasce : rinasce », d'altronde pressoché obbligate, ed il ricordo, al v. I07, della fenice che "more e poi rinasce »). In son. I99, I-II :

Io te mando, madonna, una catena d'argento e d'or, ch'al cor me fanno un laccio, aciò che tu conosci a quanto straccio l'essermi fatto servo a te me mena.

L'argento ti dimostra che ogni vena 
piena ho de fredda neve e duro giaccio ;

è foco l'or, nel qual sempre mi sfaccio

e come lui me afino in tanta pena;

quel ucel ch'è nel meggio è una phenice

che mostra el fin pur de mia vita in foco,

non come el suo, ma tristo et infelice.

evidentemente la catena regalata alla donna terminava in un medaglione effigiante, in coerenza con il topos fondamentale del fuoco amoroso, una fenice (pur arrivando a negare, per il poeta, ogni consolante ipotesi di finale rinascita: cfr. il v. II, dove el suo è riferito a el fin del v. precedente).

\section{Il pellicano}

Nel consueto contesto « disperato $:$

Ombra sotto ombra sto d'un ramo secco, assai di Erisiton più horrendo e magro, facendo un pianto sì dolente et agro che ogni fior per pietà mutato è in stecco.

Stracciomi el tristo cor e il sangue lecco, misero pelican, e in giaccio flagro più che nel fuoco ardente Meleagro, al sempre lamentar damnato di Ecco.

è inserito, ai vv. 5-6, il ricordo della leggenda del pellicano, che risuscita i suoi nati e li nutre col sangue che sgorga dal petto che esso stesso si squarcia: cfr. Phys. 4 (probabile origine di questa "natura»); CHLARO DAVANZATI V 73-74 e XII 27-28; Mare amoroso 255-62 (Poeti '200 I 497); CECCO D'AsCOLI, Acerba 2173-8I; LEONARDO, Bestiario 38 (per altri riscontri cfr. Menichetti 22).

Lo spirto salamandra

La salamandra, tradizionalmente nota per la sua capacità di sopravvivere nel fuoco, se non addirittura di estinguerlo (cfr. Phys. 3I; CHLARO DAVANZATI 28, I-4; Mare amoroso 84 [Poeti '200 I 490]; Rvf. 207, 40-4I ; CECCO 
D'ASCOLI, Acerba 2193-98; LEONARDO, Bestiario 39; e per la vasta fortuna in area romanza vedi Menichetti 23), è in un caso adibita a figurante dello spirto del poeta, in un sonetto fortemente debitore delle più tipiche, e ormai stilizzate, fisiologia e imagerie stilnovistiche :

Vola el desir fòr del mio cor dolente seguendo el spirto ch'è sua guida e scorta : questo piacer e quel speranza porta, questo dai sensi e quel vien da la mente per veder la soa luce in oriente;

ma trovando d'Amor chiusa la porta quel piange e crida e questo se conforta, l'un cade in giaccio e l'altro in foco ardente.

$\mathrm{Al}$ fin, correndo, a me tornano in fretta come agnel tristo alla penosa mandra che teme haver da' lupi acerba morte.

El cor mio, lasso, col suo spirto aspetta : si maraviglia de sì dura sorte, ché l'uno è uccel e l'altro è salamandra.

Mentre infatti (cfr. $v v .7-8)$ il desir si dispera e agghiaccia (ed è definito uccel probabilmente in quanto vola [ $[v . I]$ ), lo spirto si ricrea e infiamma in foco ardente (un'opposizione in piena regola, dunque, tra mente $e$ sensi : e cfr. il v. 4). L'intero passo (vv. I0-I4) è comunque debitore di Rvf. 207, 40-43: cfr. "mirabil salamandra» (v. 4I, hapax nei Rvf.) e il v. 43: "Felice agnello a la penosa mandra ».

La biscia superba e il serpente guardiano

L'immagine della serpe, o biscia, che annuncia l'arrivo della primavera alzando superbamente e minacciosamente il capo è singolarmente cara all'icastica attitudine figurativa e naturalistica del Sasso. Evidente, sia nelle occorrenze più «neutre » e descrittive :

Zephiro spira e col sò dolce fiato

fa l'aura intorno a sé leta e serena.

Phebo, girando, a noi la luce mena

col car de raggi più che prima ornato.

L'herbetta verde riveste ogni prato; 
con dolci versi Progne e Philomena piange, cantando la sua antiqua pena; la serpa va col capo al ciel levato.

(son. 97, I-8)

Vibra la lingua e va col capo alzato la serpe sibilante e tosicosa nella staggion ioconda et amorosa, né si aricorda del verno passato.

Quando leva le corne in ciel el Tauro et fugge la stagion cruda et acerba, leva la bissa el capo e va superba, corren le vele dal mare Indo al Mauro;

sia nell'isolata caratterizzazione etica del son. 95:

Or che sei gionta de la rota in cima, et ha sortito el tò voler effecto, non t'impir tanto di superbia il pecto che non considri el tò stato di prima.

Quel che troppo felice esser si stima non ha rason in sé, non ha intellecto, ché Fortuna crudel a il sò dilecto or li mortali abassa, or li sublima.

Amami, e 'l capo in alto non levare come serpa d'april in loco aprico, ché presto a te direbbe quel parlare :

"Quando il vilan si ritrova sul fico, tanto excellente e degno esser gli pare che non conosce parento né amico ».

è il debito da BOCCACCIO, Rime LXXVIII I-3 : "Allor che 'l regno d'Etiopia sente / il rodopeo cristallo esser deluso, / e de' sui ogni serpe leva el muso " (con l'analoga ambientazione primaverile); ma cfr. anche, con identica caratterizzazione di superbia (vedi qui, v. 3), BOLARDO, A. L. LXXXII 85 e 89-9I: " ma mentre che a le rose me apresava / [...] / scorsi una serpe de si crudel vista / che sua sembianza ancor nel cor me atrista. / Questa superba, con la testa alciata ».

Nel caso invece di son. $168,1-8$ :

Quando io vidi apparir colei che adoro corsemi el sangue, per soccorso, al core 
come dicessi : - Ecco la dea d'amore che vien, mandata dal celeste choro! Ecco che già lei tira el suo stral d'oro! Onde io rimasi como quel che, un fiore coglier vogliendo, vede un serpe fòre vibrar la lingua e minaciar martoro.

il vulgatissimo exemplum pare qui debitore (piuttosto che dei rapidi cenni di VIRGILIO, Ecl. III 93, Inf. VII 84, Rvf. 99, 5-6 e Tr. Cup. III I57) di VIRGILIO, Aen. II 379-8I, almeno per il comune diffuso compiacimento descrittivo : "Improvisum aspris veluti qui sentibus anguem / pressit bumi nitens trepidusque repente refugit / attollentem iras et coerula colla tumentem » (ma si tenga comunque presente anche l'analoga situazione, pur volta in allegoria, di BOLARDO, A. L. LXXXII \&5 ss., appena citata).

Ancora più complessa, per l'estesa allegorizzazione, è la versione di son. 28 , I-8:

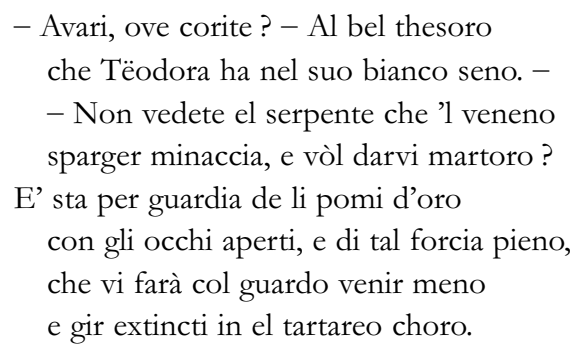

$\mathrm{Nel}$ serpente [...] che vi farà col guardo venir meno vi è il ricordo, seppure implicito, del mito di Medusa; tuttavia li pomi d'oro (v. s) sembrano indicare la contaminazione col mito delle Esperidi, già avvenuta in BOLARDO, Inn. I XII 26-4I. Nella narrazione boiardesca, infatti, erano già presenti gli elementi del «tesoro 》 (XII 27, 7-8: "et è chiamato il Tronco del Tesoro / che ha pomi de smeraldi e rami d'oro »), e del mostro visto come «serpente 》 (XII 39, 3-4: " e la sua faccia candida e vermiglia / parve di serpe terribile e fera»; II I 27, 8: "col speccbio al scudo occise quel serpente »; e vedi anche BOCCACCIO, Rime LVI $I$ : « Se quel serpente che guarda il thesoro », ripreso da GIUSTO CXLIX 35 : "Se il serpe, che guardava il mio tesoro " e soprattutto, di nuovo, da BOLARDO, A. L. LXXXII I5-I6: "Non avia visto a guardia de il tesoro / tra l'erbe il frigido angue »). Ė anche probabile la suggestione delle avventure di Orlando con la maga Falerina (Inn. II, $I V-V$ ), nel cui giardino incantato ritroviamo il dragone o "gran serpente 》 (IV I 6 ss.) e l'albero dalle " pome d'oro 》 (V 6 ss.). 


\section{Massimo Malinverni}

L'aquila e il sole

La facoltà leggendaria dell' aquila di fissare il sole (cfr. PLINIO, Nat. hist. $X$ 3, I0 ; LuCANO, Phars. IX 902-6; Phys. 6; Par. I 47-48 e XX $31-32$; Rvf. 325, 59; CECCO D'AsCOLI, Acerba 2095-2106; LEONARDO, Bestiario 36) compare nel son. 25I, dove il motivo di fondo della perdita improvisa dell'amore della donna è allegorizzato ed alluso, in ognuna delle prime tre unità metriche, in tre diversi esempi di sforzi vanamente compiuti (il coltivare in terreno sterile della prima quartina) o di felice e orgogliosa pienezza vitale ora mutata nel suo contrario per l'avversità del destino (la navigazione un tempo felice, ora perigliosa e tempestosa, della seconda quartina; il potere visivo dell'aquila specularmente converso in quello di una civetta? [notula, v. II] nella prima terzina) :

Seminai, doloroso, in nuda arena, onde sol bronchi e sterpi mieto e coglio; mutato è el dolce seme in amar loglio, doppo longe fatiche, e in secca avena.

Solcai l'onde del mar con vela piena lieto e contento : et hor con fèro orgoglio, disarmata di remi, in duro scoglio el vento irato la mia barca mena.

Como aquila guardai fisso nel sole : hor ho la vista sì debile e frale ch'io fo como la notula far sòle.

O quanto è vano ogni sperar mortale ! Chi crede a guardi, promesse e parole di donna crede al ciel volar senza ale.

I selvatici addomesticati (toro, falcone, orso, cinghiale)

Col tempo el villanel al giogo mena el tor, sì fiero e sì crudo animale ; col tempo el falcon si usa a menar l'ale e ritornar a te chiamato a pena.

Col tempo si domestica in catena el bizarro orso, el feroce cingiale ; col tempo l'aqua, che è sì molle e frale, rompe el dur sasso come el fusse arena. 


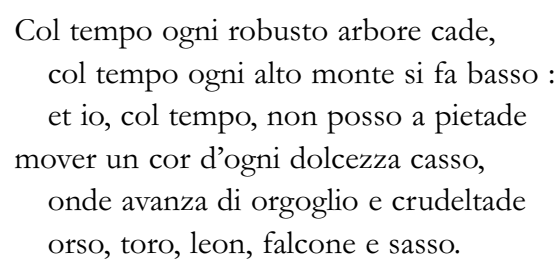

Testi di struttura e tema assai simile sono piuttosto frequenti nella lirica tardo-quattrocentesca. Vedi almeno dello stesso Sasso $i$ son. 24 I (esempio pressoché identico di combinazione di schema additivo ed anafora) e 365, il cap. XIV ("una specie di trionfo del tempo »), e lo strambotto 30 (Il tor, ch'è sì terribile animale); un sonetto del PETRUCCI (LXVI : cfr. $i$ vv. I-2: "Col tempo se conducono allo arato / li aspri tori e tornano admansati ») e soprattutto uno di JACOPO CORSI, Suole col tempo e con un poco umore (Bologna, Bibl. Universitaria, ms. 284, c. 6r; cfr. i vv. $5-8:$ : Et io, volendo intenerir un core, / trovo questi remedii esser iscarsi ; / piango, suspir, e no 'l vegio mutarsi, / ma la dureza sua farsi magiore »); quindi una serie di testi attribuibili all'AQUILANO, dal son. Col tempo passa gli anni, i mesi e l'ore (dubbie XIX), agli strambotti ${ }^{9} \mathrm{Col}$ tempo el fier caval si mette al freno; Se 'l tempo ha posto in te tanta bellezza; Se 'l tempo dona molto, el tempo toglie; Se 'l tempo spiana ogni superba alteza; Consuma el tempo ogni aspro et duro sasso. Ma per la più generale fortuna simbolica (segno di una profonda comune consonanza) di questo tema fra Quattro e Cinquecento si veda ad esempio, in campo figurativo, il Ritratto di vecchia di Giorgione (Venezia, Accademia), in cui la donna ritratta reca in mano un cartiglio con il motto, appunto, "Col tempo».

Modelli già riconosciuti per gli esempi di animali addomesticati ed elementi naturali modificati dal tempo, sia di questi versi ${ }^{10}$ come naturalmente di molte delle coeve esercitazioni sullo stesso tema testé elencate, ${ }^{\text {II }}$ sono due passi gemelli di OИIDIO : Ars am. I 47I-76 ("Tempore difficiles veniunt ad aratra iuvenci, / tempore lenta pati frena docentur equi ; / ferreus adsiduo consumitur anulus usu, / interit adsidua vomer aduncus bumo. / Quid magis est saxo durum, quid mollius unda? / Dura tamen molli saxa cavantur aqua "); ; Trist. IV VI I-I8 (cfr. vv. I-6: "Tempore ruricolae patiens fit taurus aratri, / praebet et incurvo colla premenda iugo; / tempore paret equus lentis animosus babenis, / et placido duros accipit ore lupos; / tempore Poenorum compescitur ira leonum, / nec feritas animo, quae fuit ante, manet »). 
Il delfino e Arione

Di esclusiva pertinenza mitologica è la leggenda di Arione e il delfino. Arione, poeta e citaredo di Metimna, durante un viaggio per mare dalla Sicilia verso Corinto fu salvato dalle conseguenze di un ammutinamento dei marinai da un delfino accorso al suono della sua cetra : cfr. VIRGILIO, Ecl. VIII 56; Properzio II XXVI I7-I8; OVIDIO, Fasti II 83 ss.; BocCacCIO, Rime VII 2-4 ("s'al canto d'Arion venne il delfino / facendo sé al suo legno vicino, / al suo comando presto ed ubbidiente »); BOLARDO, A. L. CIV 2-5 $e$ Inn. II XXVII I, I-4; BASINIO, Isottaeus III III 79 (e Cyris VIII I) : "pisce super curvo vectus cantabat Arion》. Tipicamente utilizzata a simboleggiare il potere divino del canto e della poesia, nel Sasso compare in una serie di testi accomunati dall'invocazione alla sonante cethra :

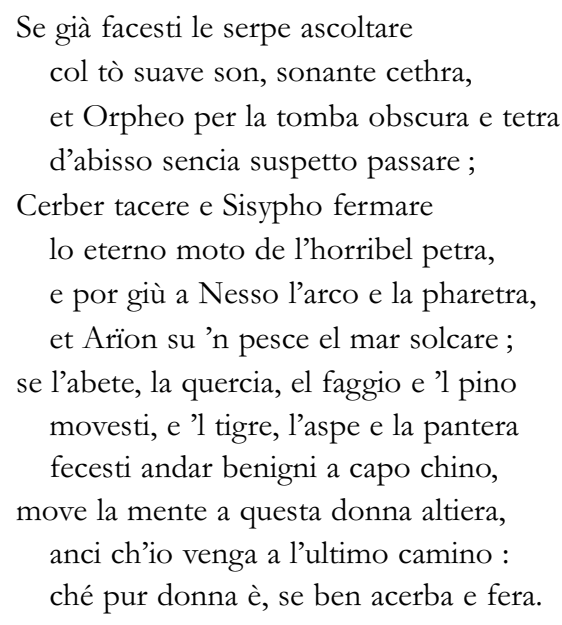

Invocazione che si preciserà, nel sonetto seguente, come lode post factum alla poesia di Serafino Aquilano (cfr. son. 92, I2 : un Saraphino), riuscita nellintento, qui solo genericamente auspicato, di muovere a compassione questa donna altiera (v. I2 : che diverrà specularmente, ad esito ottenuto, una angioletta pura) :

Fece tacer la resonante cethra

Cerbero horendo e 'l crudel Minotauro; svelse da la radice el faggio, il lauro,

specciò qual scoglio havea più dura petra.

Trasse da la spelonca obscura e tetra 
lo indomito serpente e 'l leon mauro,

e (quel che far non pò forza e thesauro)

spinse de Morte e d'Amor la pharetra.

E Thebbe e Troia circundò de mura,

fece Arïon natar sopra un delphino

e gir solcando el mar senzia paura :

maraviglia non è se un Saraphino

mossa ha col canto una angioletta pura,

ché troppo è dolce un concento divino.

(son. 92)

Una stretta affinità vi è infine col son. IOI, che riprende i miti di Anfione, Arione e Orfeo :

Cinse Amphïon cum la sua dolce lyra

Tebbe di mura e col canto suave ;

Arion de un delphin se fece nave,

e téne el vento che Neptun agira.

Compresse el docto Orpheo lo sdegno e l'ira

del re che d'Acheronte ha in man le chiave,

e firmò el sasso ponderoso e grave

che Sisipho alto e basso avolge e tira.

Tu col son de la tua arguta cethra

me facesti, con l'alma, tramutare

in una sorda, alpestra e dura petra,

et hor, vivo, me fai teco cantare :

questo è più che pasar per tomba tetra,

cinger Tebbe di mura, el mar solcare.

E per i vv. 3-4. cfr. in particolare, in questo caso, OVIDIO, Fasti II 83-84 e 97-98: " quod mare non novit, quae nescit Ariona tellus? / carmine currentes ille tenebat aquas. / [...] / forsitan, infelix, ventos undasque timebas : / at tibi nave tua tutius aequor erat».

Il basilisco

Perché io mossi da te lontano el passo altri so, forse, che mi danna e accusa e fa quel che sempre hoggi di far s'usa : cacciar quel ch'è felice al fondo, al basso. 


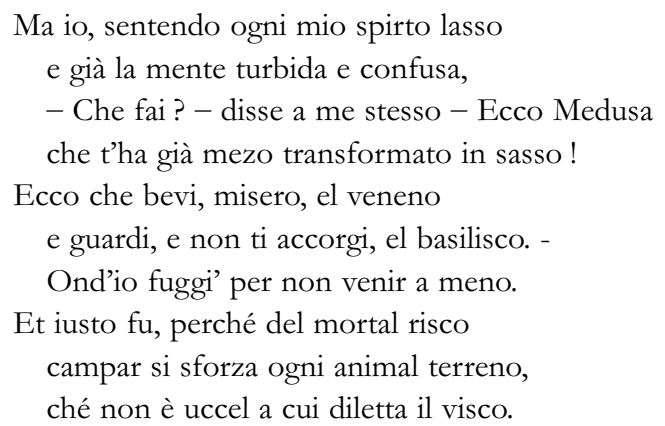

"Dai bestiari medievali proviene il motivo del basilisco, il rettile favoloso che con lo sguardo dava la morte, usato come figurante per la donna ${ }^{12}$. In realtà il tema risale a PLINIO, Nat. hist. VIII 33 e XXIX I9; LuCANO, Phars. IX 724-26; IsIDORO, Etym. XII IV 6; ma compare in seguito (per limitarsi agli esempi più notevoli della tradizione, lirica e prosastica, volgare) in GLACOMO DA LENTINI, D. 2 (Lo badalisco a lo specchio lucente, $I-2) e$ D. 3 (Guardando basalisco velenoso, I-2); STEFANO PROTONOTARO, Assai mi placeria, 42-44 (Poeti '200 I 138); Bondie DietaiUti, Madonna, me è avenuto similgliante, $3 I^{-32}$ (V I 83 ); JACOPONE DA TODI, Lauda 9a, 2 ss. (Poeti ' 200 II 9I) ; Proverbia quae dicuntur..., 469-70 (Poeti '200 I 543); Mare amoroso 94-95 (Poeti '200 I 490); CECCO D'AsCOLI, Acerba 2635-43.; FAZIO DEGLI UBERTI, Dittamondo VXVII 3 I-60; PetraRCA, Disperse XVIII s-6; PUlCI, Morgante XIV 82 e XIX 66; BolaRdo, Inn. II I 27, I-4; POLIZLANo, Rime IV 4 e CVI 25; LORENZO DE MEDICI, Canz. LIII 7-8 e CVI 7 (quindi Comento XXII 29), e Selve I 9I, 5-6 e I20, 8; LEONARDO, Bestiario I0, 50 e 75 . Sempre il Rossi segnala la particolare fortuna di questo motivo nella lirica napoletana (segnatamente in SANNAZARO, Rime XLVIII I-2 [ma vedi anche Arcadia II 94] e CARACCIOLO, Quante fiate del mio basilisco [Argo, c. 92r]) e nel più tardo ambito cortigiano (Marcello Filosseno e Olimpo da Sassoferrato). Cfr. inoltre, nel Sasso, st. 86, 5-6 (" come aquila ch'ogn'altro in vista avancia / guardava il sole, hor guardo il basilisco »), son. 373, I-2 (in contesto politico e non amoroso: "Ha partorito un basilisco el Gallo / che tutta Italia ba di veneno infusa») e soprattutto l'estesa e dettagliata similitudine di son. 374, I-8:

Ove finisse Libia è uno animale che porta il capo pel gran peso basso, col corpo infirmo, debil, stanco e lasso, e non fa con l'artiglio o il dente male, 
ma ne la vista ha un venen sì mortale ch'ogni huom che 'l mira è de la vita casso, onde molti se trovano a mal passo non estimando in lui quel che più vale.

\section{Il tristo agnello}

Simbolo sacrificale e dunque, nellimmaginario storico collettivo, animale passivo per eccellenza, l'agnello non trova certo miglior sorte nel canzoniere del Sasso. Sia che riesca a ritornare precipitosamente in seno alla mandria dopo aver temuto l'aggressione dei lupi (nel già considerato son. I33, a figurare, nei vv. 9-II, il ritorno precipitoso del « desiderio » e dello «spirito » del poeta dal vano approccio alla donna insensibile):

Al fin, correndo, a me tornano in fretta come agnel tristo alla penosa mandra che teme haver da' lupi acerba morte.

sia che si trovi solo e sperduto, lontano da gregge, madre e pastore:

$$
\begin{aligned}
& \text { Come el timido agnel del gregge fòre } \\
& \text { che se ritrova a caso abandonato, } \\
& \text { a suo modo piangendo, empie dal lato } \\
& \text { de lamenti ogni loco e di dolore } \\
& \text { quasi chiamando la matre e il pastore, } \\
& \text { cossì facc'io, dolente e sconsolato } \\
& \text { poi che, del tuo bel viso alontanato, } \\
& \text { el corpo afflitto ho sol, ché tieco è el core. }
\end{aligned}
$$

(son. I 49, I-8)

(con citazione quasi letterale da VIRGILIO, Georg. IV 5 I4-IS : "flet noctem ramoque sedens miserabile carmen / integrat et maestis late loca questibus implet ». Dunque dal lato [= dallato] sarà qui probabilmente da intendersi, in quanto tentativo di resa del virgiliano late, come "d'intorno »; e non è infine da escludere, per l'intero passo, la mediazione di Rvf. 3II, I-3: "Quel rosignuol, che si soave piagne, / forse suoi figli, o sua cara consorte, / di dolcezza empie il cielo et le campagne »), o che sia francamente atteso dal suo destino naturale, il macello, per opera del pastore (di nuovo in figura del trattamento ossessivamente riservato al poeta dall'implacabile belle dame sans merci) : 


\section{Massimo Malinverni}

Ahi, cruda serpa, hor che ligato m'hai al duro lazo, a la crudel catena, per gionger foco e fiamma a la mia pena superba, altera et in te stessa stai.

Così proprio al macel menato m'hai cum parlar dolce e cum fronte serena, come l'agnel con l'herba el pastor mena a l'ultimo suplitio de' sò guai.

Massimo Malinverni 


\section{Bestiario LIRICO}

Abbreviazioni delle opere citate:

Aquilano

Le rime di Serafino de' Ciminelli dall'Aquila, a cura di M. Menghini, Bologna i 894 (per sonetti, egloghe ed epistole).

Basinio, Cyris

Basinio Basini, Cyris, in Le poesie liriche di Basinio (Isottaeus, Cyrys, Carmina varia), a cura di F. Ferri, Torino I925.

BASINIO, Isottaeus

Basinio Basini, Liber Isottaeus, in Le poesie liriche di Basinio cit.

Boccaccio, Rime

Giovanni Boccaccio, Rime, a cura di V. Branca, in Tutte le opere, vol. 5/I (Rime, Carmina, Epistole e lettere, Vite, De Canaria, a cura di V. Branca, G. Padoan, G. Velli, G. Auzzas, R. Fabbri, M. Pastore Stocchi), Milano i 992.

Botardo, A. L.

Matteo Maria Boiardo, Amorum libri, in Opere volgari, a cura di P. V. Mengaldo, Bari i 962.

BOIARDO, Inn.

Matteo Maria Boiardo, Orlando innamorato, in Tutte le opere, I-II, a cura di A. Zottoli, Milano I936.

Caracciolo, Argo

Joan Francesco Caracciolo, Argo, in Amori de Joan Francesco Carazolo patritio neapolitano; Sonetti sextine et canzone cento del dicto poeta in laude de li ochi intitulati Argo, Napoli i so6.

Cecco D'Ascoli, Acerba

Francesco Stabili (Cecco d'Ascoli), L'Acerba, ridotta a miglior lezione ... dal prof. dott. A. Crespi, Ascoli Piceno i 927.

Chiaro Davanzati

Chiaro Davanzati, Rime, edizione critica con commento e glossario a cura di A. Menichetti, Bologna ig65 (con Menichetti si rimanda al Bestiario, alle pp. XLV-LXI dell'Introduzione; i numeri arabi si riferiscono alle singole voci).

Claudiano, De raptu Pros.

Claudio Claudiano, De raptu Proserpinae.

ERODOTO

ERoDoto, Storie.

Fazio degli Uberti, Dittamondo

Fazio degli Uberti, Dittamondo, in Il Dittamondo e le rime, a cura di G. Corsi, Bari i 95 2, 2 voll.

FEDRO

FEDro, Fabulae.

GALLO

Rime di Filenio Gallo, edizione critica a cura di M. A. Grignani, Firenze i 973. 


\section{Massimo MaLinverni}

\section{GiaCOMO DA LENTINI}

Giacomo da Lentini, Poesie, edizione critica a cura di R. Antonelli. I. Introduzione, testo, apparato, Roma i 979.

\section{Giusto}

Giusto de' Conti, Il Canzoniere, prima edizione completa a cura di L. Vitetti, Lanciano I9I 8, 2 voll.

Inf.

Dante Alighieri, La commedia. Inferno, in La commedia secondo l'antica vulgata, a cura di G. Petrocchi, Milano i966-67.

IsIDORO, Etym.

Isidori Hispalensis Episcopi Etymologiarum sive Originum libri XX. Recognovit brevique adnotatione critica instruxit W. M. Lindsay, Oxford I9I I, 2 voll.

LeOnardo, Bestiario

Leonardo da Vinci, Bestiario, in Scritti, a cura di C. Vecce, Milano i992.

Lirici toscani

Lirici toscani del Quattrocento, a cura di A. Lanza, Roma 1973-75, 2 voll.

Lorenzo de' Medici, Canz:

Lorenzo de' Medici, Canzoniere, a cura di T. Zanato, Firenze i991, 2 tomi.

Lorenzo de' Medici, Comento

Lorenzo de' Medici, Comento de' miei sonetti, a cura di T. Zanato, Firenze i 991.

Lorenzo de' Medici, Selve

Lorenzo de' Medici, Selve, in Opere, a cura di T. Zanato, Torino i 992.

Lucano, Phars.

Marco Anneo Lucano, Pharsalia.

Machiavelli, Canti carn.

Niccolo' Machiavelli, Canti carnascialeschi, in Tutte le opere, a cura di M.

Martelli, Firenze i971.

Orazio, Ars poet.

Quinto Orazio Flacco, Ars poetica.

Orazio, Carm.

Quinto Orazio Flacco, Carminum libri IV.

Ovidio, Ars am.

Publio Ovidio Nasone, Ars amatoria.

Ovidio, Fasti

Publio Ovidio Nasone, Fastorum libri VI.

Ovidio, Met.

Publio Ovidio Nasone, Metamorphoseon.

Ovidio, Trist.

Publio Ovidio Nasone, Tristium libri $V$. 


\section{BESTIARIO LIRICO}

Par.

Dante Alighieri, La commedia. Paradiso, in La commedia cit.

Petrarca, Disperse

Rime disperse di Francesco Petrarca o a lui attribuite, per la prima volta raccolte, a cura di A. Solerti, edizione postuma con prefazione, introduzione e bibliografia a cura di V. Cian, Firenze I 909.

\section{Petrucci}

E. Perito, La congiura dei baroni e il conte di Policastro, con l'edizione completa dei sonetti di G. A. de Petruciis, Bari 1926.

Phys.

Il Fisiologo, a cura di F. Zambon, Milano 1975.

Plinio, Nat. hist.

Gaio Plinio Secondo, Naturalis historia.

Poeti' 200

Poeti del Duecento, a cura di G. Contini, Milano-Napoli i 960, 2 tomi.

Poliziano, Orfeo

A. Tissoni Benvenuti, L'Orfeo del Poliziano. Con il testo critico dell'originale e delle successive forme teatrali, Padova 1986.

Poliziano, Rime

Angelo Poliziano, Rime, edizione critica a cura di D. Delcorno Branca, Firenze 1986.

\section{Properzio}

Sesto Properzio, Elegiarum libri IV.

Pulci, Morgante

Luigi Pulci, Morgante e lettere, a cura di D. De Robertis, Firenze i962.

$R v f$.

Francesco Petrarca, Canzoniere, edizione commentata a cura di M. Santagata, Milano 1996.

Sannazaro, Arcadia

Iacopo Sannazaro, Arcadia, a cura di F. Erspamer, Milano i990.

Sannazaro, Rime

Iacopo Sannazaro, Rime, in Opere volgari, a cura di A. Mauro, Bari ig6r.

SAsso, Epigr. libri

Pamphili Saxi Poetae lepidissimi Epigrammatum libri quattuor. Distichorum libri duo. De bello gallico. De laudibus Veronae. Elegiarum liber unus, Brescia I499.

Seneca, Medea

Lucio Anneo Seneca, Medea.

Tibullo

Albio Tibullo, Elegiarum libri II. 


\section{Massimo Malinverni}

\section{Tr. Cup.}

Francesco Petrarca, Triumphus Cupidinis, in Triumphi, a cura di M. Ariani, Milano I 988.

Virgilio, Aen

Publio Virgilio Marone, Aeneis.

Virgilio, Ecl.

Publio Virgilio Marone, Eclogae.

Virgilio, Georg.

Publio Virgilio Marone, Georgica. 


\section{BESTIARIO LIRICO}

I. Il Fisiologo, a cura di F. Zambon, Milano i975, pp. I3-1 5.

2. Mi sono permesso in merito qualche riflessione, blandamente provocatoria, nell'intervento al Convegno boiardesco del 1994 : cfr. La lirica volgare padana tra Boiardo e Ariosto : appunti su una transizione rimossa, in AA. Vv., Il Boiardo e il mondo estense nel Quattrocento. Atti del Convegno Internazionale di Studi. Scandiano - Modena - Reggio Emilia - Ferrara, I 3-17 settembre 1994, a cura di G. Anceschi e T. Matarrese, Padova, Antenore, I998, vol. II, pp. 695 e 7I8-ig.

3. In attesa dell'edizione, utilizzo per tutte le citazioni il testo critico da me stabilito in $I$ sonetti di Panfilo Sasso dall'editio princeps di Brescia (I50o): saggio di edirione critica e commentata, Università degli Studi di Pisa, Tesi di dottorato in Studi italianistici - V $\mathrm{V}^{\mathrm{o}}$ ciclo. Per una sintetica bibliografia ed una disamina della tradizione delle opere del modenese rimando a Sulla tradizione del sonetto «Hor te fa terra, corpo » di Panfilo Sasso, "Studi di Filologia Italiana », XLIX (1991), pp. I $23-65$.

4. A. Rossi, Serafino Aquilano e la poesia cortigiana, Brescia I980.

5. Antonio Tebaldeo, Rime della vulgata, a cura di T. Basile. II 2. Commento, Modena i992.

6. Con la sola eccezione dell'implicito accenno metaforico di son. 249, 5-8, all'interno dell'allocuzione del cagnol della donna già assunto in cielo : « Laura splendente più che 'l sol si vede, / più che 'l sol chiara e splendida Beatrice : / l'una e l'altra è qua vaga phenice / perché hebbeno del mal d'altrui mercede ».

7. È interessante osservare come nel Physiologus i capitoli sulla nottola (5) e sull'aquila (6) si susseguano direttamente.

8. A. Tissoni Benvenuti, La tradizione della terza rima e l'Ariosto, in AA. Vv., Ludovico Ariosto: lingua, stile e tradizione, Milano 1976 , p. 276.

9. Tutti editi in Bauer-Formiconi, Die Strambotti des Serafino dall'Aquila, München 1967.

ı. Cfr. J. G. Fucilla, Estudios sobre el petrarquismo en España, Madrid I960, p. 242.

i i. Cfr. il Perito in nota a Petrucci LXVI.

I 2. A. Rossi, Serafino Aquilano cit., p. 99. 